\title{
The role of vitamin $C$ in the gene expression of oxidative stress markers in fibroblasts from burn patients ${ }^{1}$
}

Jessica Bonucci', Alfredo Gragnani", Marcelo Moraes Trincado"', Victor Vincentin"', Silvana Aparecida Alves Correalv $^{\text {, Lydia Masako Ferreirav }}$

'Fellow MSc degree, Division of Plastic Surgery, Department of Surgery, Universidade Federal de São Paulo (UNIFESP), Brazil. Conception and design of the study; acquisition, analysis and interpretation of data; technical procedures; manuscript preparation and writing.

"PhD, Associate Professor, Division of Plastic Surgery, Department of Surgery, UNIFESP, Sao Paulo-SP, Brazil. Conception and design of the study; acquisition, analysis and interpretation of data; manuscript writing; critical revision; final approval.

"'Graduate student, UNIFESP, Sao Paulo-SP, Brazil. Acquisition, analysis and interpretation of data.

IVPhD, Postdoctoral degree, Division of Plastic Surgery, Department of Surgery, UNIFESP, Sao Paulo-SP, Brazil. Conception and design of the study, technical procedures, analysis and interpretation of data, statistical analysis, critical revision.

${ }^{\vee}$ Head, Full Professor, Division of Plastic Surgery, UNIFESP, Researcher 1A-CNPq, Director Medicine III-CAPES, Sao Paulo$\mathrm{SP}$, Brazil. Intellectual, scientific, conception and design of the study; critical revision.

\section{Abstract}

Purpose: To assess the action of vitamin $C$ on the expression of 84 oxidative stress relatedgenes in cultured skin fibroblasts from burn patients.

Methods: Skin samples were obtained from ten burn patients. Human primary fibroblasts were isolated and cultured to be distributed into 2 groups: TF ( $n=10$, fibroblasts treated with vitamin C) and UF ( $n=10$, untreated fibroblasts). Gene expression analysis using quantitative polymerase chain reaction array was performed for comparisons between groups.

Results: The comparison revealed 10 upregulated genes as follows: arachidonate 12-lipoxygenase (ALOX12), 24-dehydrocholesterol reductase (DHCR24), dual oxidase 1 (DUOX1), glutathione peroxidase 2 (GPX2), glutathione peroxidase 5 (GPX5), microsomal glutathione S-transferase 3 (MGST3), peroxiredoxin 4 (PRDX4), phosphatidylinositol-3,4,5trisphosphate dependent Rac exchange factor 1 (P-REX1), prostaglandin-endoperoxide synthase 1 (PTGS1), and ring finger protein 7 (RNF7).

Conclusion: Cultured fibroblasts obtained from burn patients and treated with vitamin $C$ resulted in 10 differentially expressed genes, all overexpressed, with DUOX1, GPX5, GPX2 and PTGS1 being of most interest.

Key words: Burns. Gene Expression. Fibroblasts. Oxidative Stress. Ascorbic Acid. 


\section{Introduction}

Burn injuries are a serious worldwide public health problem. More than 300.000 deaths are caused by fire-related burn injuries per year throughout the world ${ }^{1}$. Disruption in the skin integrity lead to decreased body temperature and consequent increased energy consumption. Burn injuries lead to depression of immune potential, which may result in secondary infections ${ }^{2}$. The metabolic response is immediate and may persist for at least 5 weeks. This hypermetabolic state is characterized by an increase in body temperature, oxygen and glucose consumption, carbon dioxide production, proteolysis, glycogenolysis, and lipolysis ${ }^{3}$. After a burn trauma, mediators of inflammation released at the wound site associated with cellular hydration result in extensive edema formation ${ }^{4}$.

Inflammation is an important factor in the healing of thermal injuries. The beneficial effects of local inflammation include cell growth and proliferation, protection from microbial agents and cleansing of cell debris. At the same time, a prolonged inflammatory process makes healing difficult, once the increasing on cytokines level promotes collagen degradation, dermal cell apoptosis, vascular impairment, and oxygen free radical production ${ }^{5}$. Free radicals are constantly produced during the normal functioning of cells, mostly in the form of reactive oxygen species (ROS). Reperfusion of ischemic tissues following thermal injury results in imbalance between ROS and the antioxidant defense system due to excessive generation of free radicals, known as oxidative stress $^{6}$. Studies in several organisms and cellular systems have identified the common involvement of ROS as mediators of the initial inflammatory response to wound healing?.

The defense mechanisms against oxidative stress induced by free radicals involve prevention mechanisms, repair mechanisms, physical and antioxidant defenses. Enzymatic antioxidant defenses are known as superoxide dismutase, glutathione peroxidase (GPX), and catalase. Non-enzymatic antioxidants are represented by L-ascorbic acid (vitamin C), alpha-tocopherol (vitamin E), glutathione, carotenoids, flavonoids, and other antioxidants ${ }^{8}$.

Vitamin $\mathrm{C}$ is a water-soluble micronutrient required for various biological functions. It acts as a cofactor in enzymatic reactions such as collagen synthesis ${ }^{9}$, and in human plasma is an relevant antioxidant, eliminating free radicals ${ }^{10}$. In the first 5 days after a burn injury, patients presented a decrease in vitamin C, beta-carotene, lycopene (antioxidant vitamins) and increase in lipid peroxidation products ${ }^{11}$.

An inflammatory response is triggered by a cutaneous burn with consequent production of free radicals, leading to alterations in gene expression. The identification of the mechanisms regulating gene expression of oxidative stress markers, in the presence or absence of vitamin C, is important to understand the processes that influence the recovery of burn patients ${ }^{12}$.

The purpose of the present study was to analyze the action of vitamin $C$ on the expression of 84 oxidative stress related-genes in cultured skin fibroblasts from burn patients.

\section{Methods}

This single-center, interventional, analytic, cross-sectional, controlled, in vitro study was conducted at the Plastic Surgery Unit and Burn Treatment Unit, Universidade Federal de São Paulo. It was approved by the Institutional Ethics Committee (approval number 269.015) and a written consent was given from all patients enrolled.

Ten hospitalized adult patients, consisting of 5 men and 5 women, with a 
mean age of 34.2 years, with second- or thirddegree burn injuries covering twenty five to fifty percent of the total body surface area and requiring surgical procedures were included in this study.

Patients with previous skin or superficial lesions (psoriasis and similar diseases), infectious conditions, or clinical diseases (e.g., colagenoses, such as lupus erythematosus, scleroderma, dermatopolymyositis, and mixed connective tissue disease) that could directly interfere with wound healing were not included in the study.

The " $n$ " was considered adequate because it was a lineage of cultured cells, proceeding with the extraction of its genetic material, there being no other variables besides the individual cellular genetic load. The experiment was performed after the third passage, at which time there is no cellular alteration by systemic signals that could affect the cells in the immediate isolation after obtaining the skin fragment. The sample size calculation was not performed due to lack of data in the literature explaining the number used in in vitro study, therefore, a convenience sample was used.

\section{Surgical procedure}

Debridement is the removal of dead tissue from a wound. It is commonly performed on the third day postburn in patients with a second- or third-degree burn injury. During surgical debridement, performed by the plastic surgeon, viable skin surrounding the wound is partially removed since the instrument used (Blair knife) does not allow the removal of only the necrotic skin, which has irregular margins.

These sterile fragments of normal skin that would be discarded were immediately immersed in a $50 \mathrm{~mL}$ Falcon tube containing $30 \mathrm{~mL}$ of Dulbecco's Modified Eagle's Medium (DMEM) (ATCC, New York, NY, USA) supplement with $100 \mathrm{IU} / \mathrm{ml}$ penicillin (Gibco, Big Island, NY, USA) and $100 \mu \mathrm{g} / \mathrm{ml}$ streptomycin (Gibco, Grand Island, NY, USA), then sent to the laboratory for being handled immediately.

\section{Skin fibroblasts}

Human primary fibroblasts were obtained by explant culture and distributed into 2 groups: the TF ( $n=10$, fibroblasts treated with vitamin C) and UF ( $n=10$, untreated fibroblasts) groups.

Culture flasks with some contamination or low proliferation rate (less than $80 \%$ confluence), and subsamples with insufficient extraction of RNA for analysis or loss of extracted material were excluded.

\section{Fibroblast culture medium}

The culture medium of fibroblast was composed of DMEM supplemented with antibiotics (penicillin $100 \mathrm{lU} / \mathrm{ml}$ - streptomycin/ $100 \mu \mathrm{g} / \mathrm{mL}$ ), and $20 \%$ fetal bovine serum (FBS; ATCC).

\section{Isolation and culture}

Tissue cleansing was performed within a laminar flow to maintain the sterile environment by plunging the skin fragment into 8 consecutive tubes containing $30 \mathrm{~mL}$ of modified phosphate-buffered saline (Dulbecco's PBS, without calcium and magnesium hydrochloride) (Sigma, St. Louis, USA) supplemented with $100 \mathrm{IU} / \mathrm{ml}$ penicillin (Gibco, Grand Island, NY, USA) and $100 \mu \mathrm{g} / \mathrm{ml}$ streptomycin (Gibco, Grand Island, NY, USA), shaking each tube vigorously for 45 seconds to remove blood and other impurities ${ }^{13}$.

For separation of the epidermal sheet, the skin fragments were cut into smaller pieces of $0.5 \times 0.5 \mathrm{~cm}$, placed in a $50 \mathrm{~mL}$ Falcon tube which contained $20 \mathrm{~mL}$ of dispase II (0.6-2.4 $\mathrm{U} / \mathrm{mL}$ ) (Roche Diagnostics, Indianapolis, IN) 
dissolved in DMEM, and maintained for $18 \mathrm{~h}$ in the refrigerator. The fragments were washed to neutralize the dispase with Versene (Gibco) and then the dermis and the epidermis were separated mechanically on a $100 \mathrm{~cm}^{2}$ Petri dish.

For fibroblast isolation, the dermis pieces were placed on a $100-\mathrm{cm}^{2}$ Petri dish with small cuts made with a scalpel blade. After $20 \mathrm{~min}$, when the fragments were adhered to the dish, was added $10 \mathrm{~mL}$ of fibroblast culture medium. The cells reached $80 \%$ confluence after about 7 days of culture on the Petri dish. Then, the fibroblasts were trypsinized and transferred to $75-\mathrm{cm}^{2}$ culture flasks. Third-passage fibroblasts were either used in the analysis or frozen in liquid nitrogen until required.

\section{Vitamin C supplementation}

In the TF group ( $\mathrm{n}=10)$, vitamin $\mathrm{C}$ (L-ascorbic acid; Sigma, St. Louis, MO) was added at a concentration of $250 \mu \mathrm{M}$ in $10 \mathrm{ml}$ of fibroblast culture medium. The solution was prepared immediately prior to use and care was taken to protect it from light, once vitamin $\mathrm{C}$ is photosensitive. The supplemented medium with vitamin $C$ was placed in the flask along with the cells in the third passage and then changed after 48 hours. On the fourth day, which was the time expected for cells to fulfill $80 \%$ confluence in the flask, the cells were removed. The solution $\mathrm{pH}$ was maintained close to 7.4 .

\section{RNA isolation and purification}

Total RNA was extracted from cells using Trizol reagent (Invitrogen, Foster City, CA) and isolated with the ArrayGrade Total RNA Isolated Kit (SABiosciences, Frederick, MD), that is a silica membrane that filters and retains the RNA during centrifugation, providing elevated yield and purity. A buffer stabilized the RNA, preventing its degradation and retaining it in the spin column.

After the complete dissociation of nucleoprotein complexes, phase separation was obtained with chloroform (Sigma) and centrifugation at $10.000 \mathrm{rpm}$ for $5 \mathrm{~min}$. The precipitated RNA was washed in $75 \%$ ethanol, and redissolved in RNase-free water. It was used the RNeasy Mini kit (Qiagen, Valencia, CA) to purify Total RNA and then treated with DNAse. The quality and the amount of the material extracted were obtained using a bioanalyzer (Agilent Technologies Inc., Palo Alto, CA) and a spectrophotometer - NanoVue GE Plus (GE Healthcare, Cleveland, $\mathrm{OH}$ ).

Quantitative polymerase chain reaction ( $q P C R$ ) array

The qPCR array was performed in a thermal cycler with an optical system for fluorescence excitation and emission detection coupled to a computer with software for data acquisition and analysis. It uses the RNA molecule as an initial template and, by reverse transcription, generates the complementary DNA (cDNA) from triphosphate nucleotides, primers, initial sequences for DNA polymerization, and DNA polymerase by cyclic amplification, which results in cDNA copies. Fluorescence values are recorded during each cycle and correspond to the amount of amplified product, represented by a sigmoid curve ${ }^{12,14}$.

The quantification cycle $(\mathrm{Cq})$, also known as threshold cycle, take-off point or crossing point, is the cycle number at which the reaction reaches the maximum and minimum thresholds during the exponential phase. It allows the exact and reproducible quantification of the cycles. The relative expression of the qPCR array combines relevant $\mathrm{Cq}$ values, or their mean values, through a mathematical equation, resulting in the relative expression ratio or fold change 
between the sample and control.

In this study, $1 \mu \mathrm{g}$ of total RNA from each sample was used per array plate for CDNA synthesis. The RT ${ }^{2}$ First Strand kit (SABioscience Corporation, Fredick, MD) was utilized for reverse transcription reactions and complete removal of genomic DNA. For the qPCR array analysis, it was used the RT2 Profiler PCR Array System (SABioscience), which contained a plate with 84 genes. Each of the 84 genes composed on the plate is already known to be related to oxidative stress.

The amplification, acquisition, and analysis of the data were performed using a real-time thermal cycler or qPCR array $(A B I$ Prism 7500 Sequence Detection System, Applied Biosystems, Foster City, CA). The analyses were carried out with pooled cDNA from all patients in each group and performed in triplicate for sample variability.

\section{Statistical analysis}

Data analysis of qPCR array was executed by RT2 Profiler PCR Array Data Analysis Web Portal (http://pcrdataanalysis. sabiosciences.com/pcr/arrayanalysis.php).

Nonparametric Friedman's test was used for multiple comparisons and the nonparametric Wilcoxon test for paired variables was applied to evaluate differences between measurements. All statistical tests were performed at a significance level $\alpha$ of $0.05(p<0.05)$.

\section{Results}

Skin samples for RNA extraction and protein expression analysis were obtained from 5 men and 5 women, with a mean age of $34.2 \pm 10.2$ years, who were included in the study.

The comparison of gene expression between the TF ( $n=10$; fibroblasts treated with vitamin C) and UF ( $\mathrm{n}=10$; untreated fibroblasts) groups revealed that $11.9 \%(n=10)$ of the 84 genes used as markers of oxidative stress were significantly (Table 1). No downregulated gene was found. For all comparisons, the values from the UF group were used as a reference.

Table 1 - Genes differentially expressed in treated fibroblasts compared to untreated fibroblasts.

\begin{tabular}{ccc}
\hline Upregulated genes & Fold change & $\boldsymbol{p}$-value \\
\hline ALOX12 & 2.29 & $0.0323^{*}$ \\
DHCR24 & 2.19 & $0.0453^{*}$ \\
DUOX1 & 3.97 & $0.0048^{*}$ \\
GPX2 & 2.13 & $0.0282^{*}$ \\
GPX5 & 4.80 & $0.0125^{*}$ \\
MGST3 & 2.25 & $0.0021^{*}$ \\
PRDX4 & 2.40 & $0.0372^{*}$ \\
P-REX1 & 5.11 & $0.0418^{*}$ \\
PTGS1 & 9.65 & $0.0044^{*}$ \\
RNF7 & 3.38 & $0.0027^{*}$ \\
\hline
\end{tabular}

Asterisks $\left({ }^{*}\right)$ indicate statistical significance $(p<0.05)$. ALOX12, arachidonate 12-lipoxygenase; DHCR24, 24-dehydrocholesterol reductase; DUOX1, dual oxidase 1; GPX2, glutathione peroxidase 2; GPX5, glutathione peroxidase 5; MGST3, microsomal glutathione S-transferase 3; PRDX4, peroxiredoxin 4; P-REX1, phosphatidylinositol-3,4,5-trisphosphate dependent Rac exchange factor 1; PTGS1, prostaglandin-endoperoxide synthase $1 ;$ RNF7, ring finger protein 7.

The heat map for comparisons between groups is shown in Figure 1. The green color represents the downregulated genes, while the red color represents the upregulated genes. The color near the black shows genes without significant gene expression, and the gray color represents the non-expressed genes.

The scatter plot is shown in Figure 2. It presents the fold change value of the relative gene expression of the analyzed group in relation to the reference group. The central line represents the gene expression of the reference or control group, while the points 
reveal fold change values of the group under analysis. The other lines, above and below the centerline, delimit fold change, equal to two, with points above the upper line appearing green representing upregulated genes and points that extend beyond the bottom line, which appear in red, reveal downregulated genes. Dots that appear within lines, with fold change, or between 2 and -2 , appear in colorations close to black and represent genes with no difference in relative gene expression.
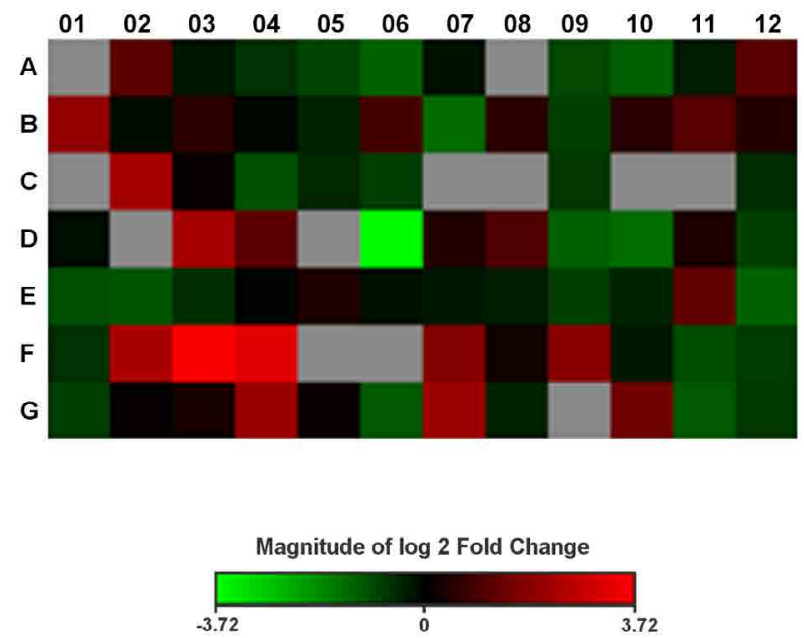

Figure 1 - Heat map of log 2 fold change values for the treated fibroblast (TF) group versus untreated fibroblast (UF) group.

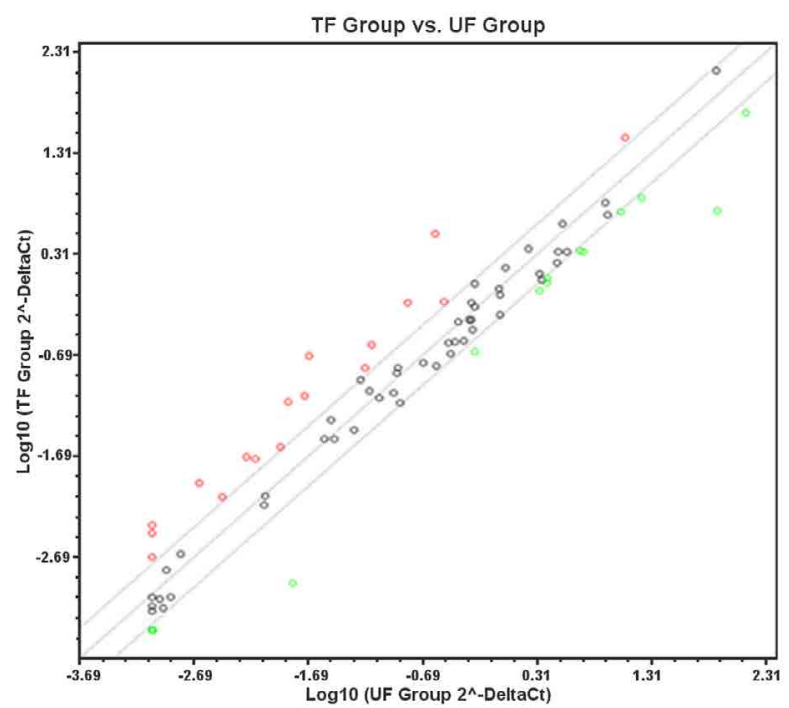

Figure 2 - Scatter plot showing the relative gene expression between the treated fibroblast (TF) group and untreated fibroblast (UF) group.

\section{Discussion}

A previous study in burn patients has identified a transcriptome profile of genes that initiate and maintain systemic inflammation in an oxidative stress environment ${ }^{15}$. Thermal injury mediates a common systemic response, independent of the site or extent of injury, and specific responses to local and distant trauma. Other studies identified oxidative stressrelated genes differentially expressed in groups of burn patients ${ }^{16,17}$

This original study assessed the action of vitamin $C$ on the expression of 84 oxidative stress related-genes in cultured skin fibroblasts from burn patients using qPCR array and identified a gene expression profile that will serve as a basis for further investigations.

genes were identified as follows: arachidonate 12-lipoxygenase (ALOX12), 24-dehydrocholesterol reductase (DHCR24), dual oxidase 1 (DUOX1), glutathione peroxidase 2 (GPX2), glutathione peroxidase 5 (GPX5), microsomal glutathione S-transferase 3 (MGST3), peroxiredoxin 4 (PRDX4), phosphatidylinositol-3,4,5-trisphosphate dependent Rac exchange factor 1 (P-REX1), prostaglandin-endoperoxide synthase 1 (PTGS1), and ring finger protein 7 (RNF7). Four of the 10 genes are related to oxidative stress, while 6 genes are associated with antioxidants.

ALOX12 (fold change $=2.29 ; p=0.0323$ ) is a lipoxygenase that contributes to the epidermal barrier function. When a decrease in the intracellular levels of the antioxidant glutathione occurs, ALOX12 becomes neurotoxic and amplifies the oxidative stress by attacking the mitochondria, leading to the production of ROS ${ }^{18}$.

DHCR24 (fold change $=2.19 ; p=0.0453$ ) is a multifunctional, endoplasmic reticulumresident enzyme that exhibits anti-apoptotic activity and is involved in cholesterol synthesis. 
It exerts cytoprotective effects against endoplasmic reticulum stress by eliminating ROS. DHCR24 can scavenge hydrogen peroxide $\left(\mathrm{H}_{2} \mathrm{O}_{2}\right)$, protecting cells from oxidative stressinduced apoptosis ${ }^{19}$.

DUOX1 (fold change $=3.97 ; p=0.0048$ ) encodes a glycoprotein from the nicotinamide adenine dinucleotide phosphate (NADPH) oxidase family. NADPH oxidase plays an important role in the innate immune response to infection or injury, which is characterized by the production of $\mathrm{H}_{2} \mathrm{O}_{2}$. It also participates in autocrine and paracrine signaling mechanisms that regulate proinflammatory and wound healing responses. Recent studies have indicated that $\mathrm{H}_{2} \mathrm{O}_{2}$-dependent $\mathrm{NADPH}$ oxidases (NOX) and Dual oxidases (DUOX) may be involved in paracrine signaling, recruiting neutrophils and macrophages to the wound site ${ }^{7}$.

GPX2 (fold change $=2.13 ; p=0.0282$ ) is a member of the glutathione peroxidase family and encodes a selenium-dependent glutathione peroxidase enzyme, which is one of the two isoenzymes responsible for most $\mathrm{H}_{2} \mathrm{O}_{2}$ reduction. Decreased expression of Forkhead box M1 (FOXM1), a member of a transcription factor superfamily, is sufficient to induce keratinocyte senescence combined with increased production of ROS and inhibition of ROS-scavengers, including GPX2, PRDX, superoxide dismutase 2 (SOD2), and catalase. FOXM1 depletion sensitizes normal keratinocytes to apoptosis and ROS-induced apoptosis. This characterizes FOXM1 as a key regulator of ROS in dividing normal epithelial cells ${ }^{20}$.

GPX5 (fold change $=4.80 ; p=0.0125$ ) belongs to the glutathione-peroxidase family and is expressed in the reproductive tract of male mammals and regulated by androgen. Oxidative stress in epididymal cell culture has been studied as a function of the GPX5 gene expression. The authors reported that the action of the protein encoded by GPX5 is protective against the damaging effects of $\mathrm{H}_{2} \mathrm{O}_{2}$ to which the spermatozoa are exposed ${ }^{21}$.

MGST3 (fold change $=2.25 ; p=0.0021$ ) encodes a member of the membrane associated proteins in eicosanoid and glutathione metabolism (MAPEG) family. Members of this family are related with the production of leukotrienes and prostaglandins, which are important mediators of inflammation. This enzyme also shows a glutathionedependent peroxidase activity toward lipid hydroperoxide ${ }^{22}$.

PRDX4 (fold change $=2.40 ; p=0.0372$ ) encodes a protein, which is an antioxidant enzyme. PRDX are a family of enzymes that uses thioredoxin (Trx) and other electron donors for the reduction of peroxide. Many small molecules and proteins, such as glutathione and Trx, are involved in redox reactions. The involvement of PRDX in hydrogen peroxide-mediated signaling has become evident with the better understanding of the signaling roles of hydrogen peroxide in response to extracellular stimuli. Signal regulation by ROS, particularly $\mathrm{H}_{2} \mathrm{O}_{2}$, seems to be the most profound function of PRDX. PRDX4 is the only member of the PRDX family showing a hydrophobic signal peptide at the amino terminus, thus exhibiting the ability to undergo either secretion or retention by the endoplasmic reticulum, which is the organelle that produces the highest levels of $\mathrm{H}_{2} \mathrm{O}_{2}$, the major source of ROS ${ }^{23}$.

P-REX1 (fold change $=5.11 ; p=$ 0.0418 ) encodes a protein that acts as a guanine nucleotide exchange factor and belongs to the Rho family of small Rho guanosine triphosphatase (GTPase) binding proteins, called Rac proteins. P-REX1 is found primarily in the cytoplasm and activated by phosphatidylinositol-3,4,5-trisphosphate and 
the beta-gamma subunits of heterotrimeric $\mathrm{G}$ proteins. It is involved in endothelial activation, enhancing the production of oxidizing agents, and therefore increasing capillary permeability, which contributes to tissue edema, hemostatic actions in platelet aggregation, activation of endothelial growth factors and angiogenesis, and bactericidal function of leukocytes ${ }^{24}$.

PTGS1 (fold change $=9.65 ; p=0.0044$ ) is a gene whose expression involves enzymes that catalyze the conversion of arachidonic acid to prostaglandin. Its encoded protein, known as cyclooxygenase (COX), regulates angiogenesis in endothelial cells, and COX-1 and COX-3 participate in the inflammatory response. PTGS1 (COX-1) is the main source of endothelial prostacyclin (PGI2), which has been described as a vasodilator in cultured cells under altered oxidative stress ${ }^{25}$. Our results may be relevant for the reassessment of the impact of COX inhibitors on the production of vascular PGI2 in patients experiencing significant oxidative stress.

RNF7 (fold change $=3.38 ; p=0.0027$ ) encodes a highly conserved protein, which is an essential subunit of the S-phase kinaseassociated protein 1 (SKP1)-Cullin/cell division cycle 53 (CDC53)-F box protein complex, also known as the SCF complex. It is part of a protein degradation mechanism, which plays an important role in cell cycle progression and signal transduction. This protein is sensitive to oxidative stress and protects mammalian cells against apoptosis, as it is an intracellular antioxidant molecule that may act as a redox sensor, buffering cells against oxidative-stress induced damage ${ }^{26}$.

Vitamin $C$ has shown to be effective in the treatment burn patients due to its pro- and antioxidant properties. The initial hypothesis that vitamin C could alter the expression of oxidative stress-related genes in cultured skin fibroblasts from patients with severe burn was confirmed. The comparison of gene expression between fibroblasts treated with vitamin C and untreated fibroblasts revealed that 10 genes used as markers of oxidative stress were significantly upregulated.

\section{Conclusion}

Cultured skin fibroblasts obtained from burn patients and treated with vitamin C resulted in 10 differentially expressed genes, all overexpressed, with DUOX1, GPX5, GPX2 and PTGS1 being of most interest.

\section{References}

1. Mock C, Peck M, Krug E, Haberal M. Confronting the global burden of burns: a WHO plan and a challenge. Burns. 2009;35:615-7. doi: 10.1016/j. burns.2008.08.016.

2. Spanholtz TA, Theodorou P, Amini P, Spilker G. Severe burn injuries: acute and long-term treatment. Dtsch Arztebl Int. 2009;106:60713. doi: 10.3238/arztebl.2009.0607.

3. Parihar A, Parihar MS, Milner S, Bhat S. Oxidative stress and anti-oxidative mobilization in burn injury. Burns. 2008;34:617. doi: 10.1016/j.burns.2007.04.009.

4. Jeschke MG, Mlcak RP, Finnerty CC, Norbury WB, Gauglitz GG, Kulp GA, Herndon DN. Burn size determines the inflammatory and hypermetabolic response. Crit Care. 2007;11:R90. doi: 10.1186/cc6102.

5. Shupp JW, Nasabzadeh TJ, Rosenthal DS, Jordan $\mathrm{MH}$, Fidler $\mathrm{P}$, Jeng JC. A review of the local pathophysiologic bases of burn wound progression. J Burn Care Res. 2010;31:84973. doi: 10.1097/BCR.0b013e3181f93571.

6. Carden DL, Granger DN. Pathophysiology of ischaemia reperfusion injury. J Pathol. 2000;190:255-66. doi: 10.1002/ (SICI)1096-9896(200002)190:3<255::AIDPATH526>3.0.CO;2-6

7. Hristova M, Veith $C$, Habibovic A, Lam YW, Deng $B$, Geiszt $M$, Janssen-Heininger $Y M$, van der Vliet A. Identification of DUOX1dependent redox signaling through protein 
S-glutathionylation in airway epithelial cells. Redox Biol. 2014;15:436-46. doi: 10.1016/j. redox.2013.12.030.

8. Valko M, Leibfritz D, Moncol J, Cronin MT, Mazur M, Telser J. Free radicals and antioxidants in normal physiological functions and human disease. Int J Biochem Cell Biol. 2007;39:44-84. doi: 10.1016/j. biocel.2006.07.001.

9. Darr D, Combs S, Pinnell S. Ascorbic acid and collagen synthesis: rethinking a role for lipid peroxidation. Arch Biochem Biophys. 1993;307:331-5. doi: 10.1006/ abbi.1993.1596.

10.Frei B, Stocker R, Ames BN. Antioxidant defenses and lipid peroxidation in human blood plasma. Proc Natl Acad Sci USA. 1988;85:9748-52. PMID: 3200852

11.Bertin-Maghit $M$, Goudable J, Dalmas E, Steghens JP, Bouchard C, Gueugniaud PY, Petit P, Delafosse B. Time course of oxidative stress after major burns. Intensive Care Med. 2000;26:800-3. doi: 10.1007/ s001340051250.

12.Sanders R, Mason DJ, Foy CA, Huggett JF. Considerations for accurate gene expression measurement by reverse transcription quantitative PCR when analysing clinical samples. Anal Bioanal Chem. 2014;406:647183. doi: 10.1007/s00216-014-7857-x.

13.Manuel J, Gragnani A, Ferreira LM. Ácido ascórbico intracelular diminui a indução à senescência celular, mas não a apoptose em fibroblastos expostos a concentrações subtóxicas de H2O2. Rev Bras Queimaduras. 2012;11:56-62.

14.Bustin SA. Why the need for qPCR publication guidelines?--The case for MIQE. Methods. 2010;50:217-26. doi: 10.1016/j. ymeth.2009.12.006.

15.Padfield KE, Zhang Q, Gopalan S, Tzika AA, Mindrinos MN, Tompkins RG, Rahme LG. Local and distant burn injury alter immunoinflammatory gene expression in skeletal muscle. J Trauma. 2006;61:280-92. doi: 10.1097/01.ta.0000230567.56797.6c

16.Nguyen TT, Gilpin DA, Meyer NA, Herndon DN. Current treatment of severely burned patients. Ann Surg. 1996;223:14-25. PMID: 8554414.

17.Jordana $M$, Särnstrand $B$, Sime PJ, Ramis I. Immune-inflammatory functions of fibroblasts. Eur Respir J. 1994;7:2212-22. PMID: 7713206.

18.Pallast S, Arai K, Wang $\mathrm{X}$, Lo $\mathrm{EH}$, van Leyen K. 12/15-Lipoxygenase targets neuronal mitochondria under oxidative stress. J Neurochem. 2009;111:882-9. doi: 10.1111/j.1471-4159.2009.06379.x.

19.Lu X, Kambe F, Cao X, Kozaki Y, Kaji T, Ishii T, Seo $H$. $3 \beta$-Hydroxysteroid- $\Delta 24$ reductase is a hydrogen peroxide scavenger, protecting cells from oxidative stress-induced apoptosis. Endocrinology. 2008;149:326773. doi: 10.1210/en.2008-0024.

20.Smirnov A, Panatta E, Lena A, Castiglia D, Di Daniele N, Melino G, Candi E. FOXM1 regulates proliferation, senescence and oxidative stress in keratinocytes and cancer cells. Aging (Albany NY). 2016;8:1384-97. doi: 10.18632/aging.100988.

21.Vernet $P$, Rigaudiére $N$, Ghyselinck $N$, Dufaure JP, Drevet JR. In vitro expression of a mouse tissue specific glutathioneperoxidase-like protein lacking the selenocysteine can protect stably transfected mammalian cells against oxidative damage. Biochem Cell Biol. 1996;74:125-31. PMID: 9035686.

22. Nebert DW, Vasiliou V. Analysis of the glutathione S-transferase (GST) gene family. Hum Genomics. 2004;1:460-4. doi: 10.1186/1479-7364-1-6-460.

23.Fujii J, Ikeda $\mathrm{Y}$, Kurahashi $\mathrm{T}$, Homma $\mathrm{T}$. Physiological and pathological views of peroxiredoxin 4. Free Radic Biol Med. 2015;83:373-9. doi: 10.1016/j. freeradbiomed.2015.01.025.

24.Carretero-Ortega J, Walsh CT, HernándezGarcía R, Reyes-Cruz G, Brown JH, Vázquez-Prado J. Phosphatidylinositol 3,4,5-triphosphate-dependent Rac exchanger 1 (P-Rex-1), a guanine nucleotide exchange factor for Rac, mediates angiogenic responses to stromal cellderived factor-1/chemokine stromal cell derived factor-1 (SDF-1/CXCL-12) linked to Rac activation, endothelial cell migration, and in vitro angiogenesis. Mol Pharmacol. 2010;77:435-42. doi: 10.1124/ mol.109.060400.

25.Toniolo A, Buccellati C, Pinna C, Gaion RM, Sala A, Bolego C. Cyclooxygenase-1 and prostacyclin production by endothelial cells 
in the presence of mild oxidative stress. PLoS One. 2013;8:256683. doi: 10.1371/ journal.pone.0056683.

26.Swaroop $M$, Bian J, Aviram $M$, Duan $H$, Bisgaier $C L$, Loo JA, Sun Y.
Expression, purification, and biochemical characterization of SAG, a ring finger redoxsensitive protein. Free Radic Biol Med. 1999;27:193-202. doi: 10.1016/S08915849(99)00078-7.

\section{Correspondence:}

Alfredo Gragnani

Rua Pedro de Toledo, $720 / 2^{\circ}$ andar

04024-002 São Paulo - SP Brasil

Tel.: (55 11)5576-4848

agragnanif@gmail.com

Received: Apr 28, 2018

Review: June 25, 2018

Accepted: July 23, 2018
Conflict of interest: none

Financial source: FAPESP (number 2013/10905-0)

${ }^{1}$ Research performed at Division of Plastic Surgery, Department of Surgery, Universidade Federal de São Paulo (UNIFESP), Brazil. 